\title{
Memory guided saccade deficit after caudate nucleus lesion
}

\author{
A-I Vermersch, B M Gaymard, S Rivaud-Pechoux, C J Ploner, Y Agid, \\ C Pierrot-Deseilligny
}

\begin{abstract}
The role of the caudate nucleus in ocular motor control is not well determined in humans. Eye movements were recorded from a 45 year old man with infarctions involving bilaterally the body of the caudate nucleus, with a greater extent on the left side. The patient exhibited a pattern of eye movement abnormalities in which a delay dependent decrease of accuracy of memory guided saccades predominated. By contrast, memory guided pointing was normal. It is concluded that the body of the caudate nucleus participates in a spatial short term memory network devoted to eye movements.
\end{abstract}

(F Neurol Neurosurg Psychiatry 1999;66:524-527)

Keywords: saccade deficit; caudate nucleus lesion

Parietal and prefrontal cortices play a crucial role in tasks in which a movement must be directed towards the memorised location of a visual target. ${ }^{12}$ In monkeys, electrophysiological studies have shown spatially selective neuronal delay activity in the dorsolateral prefrontal cortex (dlPFC), ${ }^{3}$ the frontal eye field $(\mathrm{FEF}),{ }^{45}$ and the posterior parietal cortex (PPC). ${ }^{6}$ Subcortical structures are also involved in this network. Neurons in the caudate
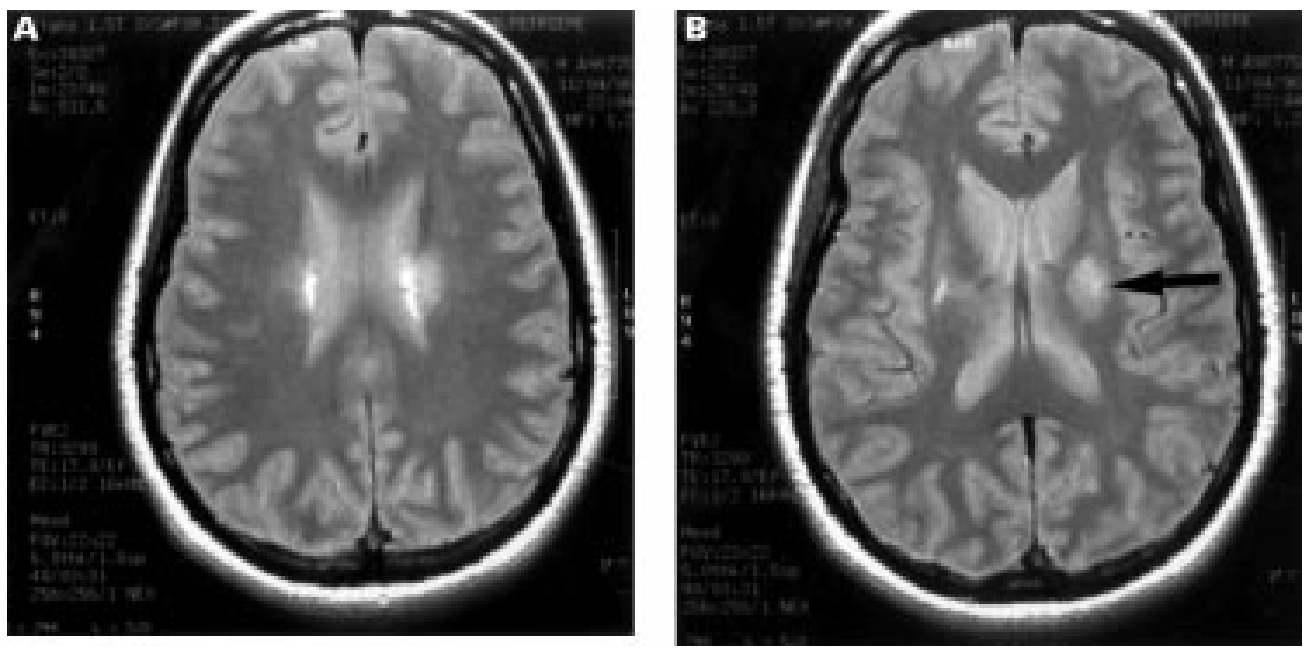

Figure 1 Bilateral striatal lesions (T2 weighted axial MRI), larger on the left side. The lesions involved the body of the caudate nucleus $(A)$ and the posterior part of the putamen (B, arrow), but spared the head of the caudate nucleus $(B)$.

nucleus $(\mathrm{CN})$ and in the substantia nigra pars reticulata (SNpr) respond during spatial memory encoding ${ }^{78}$ and during maintenance of a spatial stimulus across a delay. ${ }^{7-10}$ Therefore, spatial short term memory seems to involve a network including cortical (PPC, dlPFC, FEF) and subcortical (CN and SNpr) structures.

In humans, lesion studies have confirmed the role of the PPC, dlPFC, and FEF in the control of memory guided saccades, ${ }^{11}$ but the role of the $\mathrm{CN}$ in such tasks has up to now not been investigated. We studied a patient with a bilateral caudate lesion, with an emphasis on spatial short term memory paradigms; memory guided saccades and memory guided pointing tasks were tested with different memorisation delays. To compare the pattern of ocular motor impairments with that found after cortical frontal lesions, visually guided saccades and antisaccades were also studied.

\section{Case report}

A 45 year old right handed man, with a history of hypertension, was admitted after sudden onset of a right sided hemiparesis and dysarthria that resolved totally within 20 minutes. On MRI, an ischaemic lesion was found in the left striatum (fig $1 \mathrm{~A}$ and B), involving the body (fig $1 \mathrm{~A}$ ) but not the head (fig $1 \mathrm{~B}$ ) of the $\mathrm{CN}$

France

A-I Vermersch

B M Gaymard

$S$ Rivaud-Pechoux

C J Ploner

Y Agid

C Pierrot-Deseilligny

Klinik für Neurologie, Charité,

Humboldt-Universität Berlin, Berlin,

Germany

C J Ploner

Correspondence to:

Dr A-I Vermersch, INSERM U 289, Hôpital de la

Salpêtrière,

47 Boulevard de l'Hôpital,

75651 Paris Cedex 13,

France.

Received 8 July 1998 and in revised form

22 September 1998

Accepted 23 September 1998 
and the posterior part of the putamen (fig $1 \mathrm{~B}$ ). In the right hemisphere, a symmetric, although much smaller lesion, involved the same structures (fig $1 \mathrm{~A}$ and B). Eye movements were recorded 51 days after the stroke. At the time of eye movement recording, neurological examination was normal. Visual field and visual acuity were normal, and eye movements were conjugate and of full range.

An age matched control group of eight normal subjects (four women, four men, mean age 42 (SD 5.5) years) was also studied with the same paradigms as the patient. Control subjects were normal on neurological examination. Informed consent was obtained from the patient and all control subjects.

\section{Materials and methods}

Eye movements were recorded by direct current electro-oculography, in complete darkness with bitemporal electrodes. ${ }^{12}$

\section{VISUALLY GUIDED SACCADES}

In this task, the central fixation point was presented for 2 to 4 seconds, than switched off 200 $\mathrm{ms}$ (gap) before the onset of a luminous lateral target ( 1 second) located $25^{\circ}$ to the right or left of the central fixation point. The subject was instructed to fixate the central fixation point and to move his eyes to the lateral target as soon as it appeared. The target was presented pseudorandomly right or left, with unpredictable timing. Left and right saccade latencies were calculated by averaging 20 measurements in each direction. Saccade accuracy was expressed as saccade gain (amplitude of the first saccade over target eccentricity).

\section{ANTISACCADES}

The stimulation was the same as in the gap task except that the subject was instructed to move his eyes immediately in the direction opposite to the lateral target. The percentage of errors (misdirected saccades - that is, initial saccades directed towards the target) was determined for each direction.

\section{MEMORY GUIDED SACCADES}

Memory guided saccades were studied with three different memorisation delays. While subjects fixated a central fixation point, a single lateral target was flashed for $50 \mathrm{~ms}$, pseudorandomly right or left, with unpredictable eccentricity $\left(10^{\circ}, 15^{\circ}, 20^{\circ}, 25^{\circ}\right.$, or $\left.30^{\circ}\right)$. Subjects were instructed to maintain their eyes on the central fixation point during the memorisation delay. In some blocks of trials, this delay lasted 1 second, in others 2 seconds, and in others 7 seconds. Central fixation point offset was the go signal for a saccade directed to the memorised location of the flash. Then, the lateral target was reilluminated to allow a corrective saccade, if necessary. For each delay, at least 15 saccades in each direction were recorded. Right and left saccade latencies were calculated and gain of the first saccade and of the final eye position - that is, the eye position reached just before reillumination of the lateral target.
MEMORY GUIDED POINTING TASK

The same stimulation as in the memory guided saccade task was used, except that the subject was requested to point with the hand to the remembered location of the flash without moving the eyes. The right arm was studied for rightward targets and the left arm for leftward targets. Subjects closed their eyes after reaching the target, then a spotlight, focused on the ramp, was turned, and the position of the index finger on a grid adjacent to the light emitting diodes was recorded. Only a 7 second delay was used. Pointing accuracy was expressed as final gain.

SEQUENCE OF PARADIGMS

In all subjects, the same following sequence of paradigms was realised. In a first session, the gap task was performed, then the memory guided saccade task with a 7 second and a 2 second delay and, finally, the antisaccade task. During a second recording session (after a break of several hours), the memory guided pointing task (7 second delay) and the memory guided saccade task with a 1 second delay were performed.

\section{STATISTICS}

Mean values of all parameters were calculated both in the patient and controls. Patient values above or below 2 SDs of the mean values of controls were considered as abnormal.

\section{Results}

In our patient, mean latency of visually guided saccades was $208 \mathrm{~ms}$ rightwards and $183 \mathrm{~ms}$ leftwards, which was within $2 \mathrm{SD}$ of controls (R: 110- $230 \mathrm{~ms}, \mathrm{~L}: 83-219 \mathrm{~ms}$ ). Furthermore, leftward visually guided saccade gain (0.93) was within 2 SDs of controls (0.91-0.99). Rightward visually guided saccade gain (0.82) was below 2 SD of controls (0.92-1).

In our patient, mean latency of antisaccades was 339 ms rightwards and 289 ms leftwards, which was within 2 SD of controls (R: 189-345 $\mathrm{ms}, \mathrm{L}: 167-347 \mathrm{~ms})$. The percentage of misdirected saccades in the antisaccade task was $0 \%$ rightwards and 9\% leftwards, and thus analogous to mean values of controls (R: $9 \%$, L: $13 \%)$.

Mean latency of memory guided saccades was $414 \mathrm{~ms}$ leftwards, within $2 \mathrm{SD}$ of controls (166-426 ms). By contrast, mean latency of rightward memory guided saccades was 469 ms - that is, above 2 SD of controls (171-423 $\mathrm{ms})$. All gains were decreased in the memory guided saccade paradigm, with the decrease depending on the duration of the delay. Mean gain of first saccades was 0.77 rightwards and 0.74 leftwards with a 1 second delay, 0.46 rightwards and 0.62 leftwards with a 2 second delay and, lastly, 0.38 rightwards and 0.55 leftwards with a 7 second delay. Mean gain of final eye positions was 0.78 rightwards and 0.88 leftwards with a 1 second delay, 0.48 rightwards and 0.76 leftwards with a 2 second delay and lastly, 0.39 rightwards and 0.62 leftwards with 7 second delay. All memory guided saccade gains were below $2 \mathrm{SD}$ of controls (fig 2), except for the rightward first saccade 

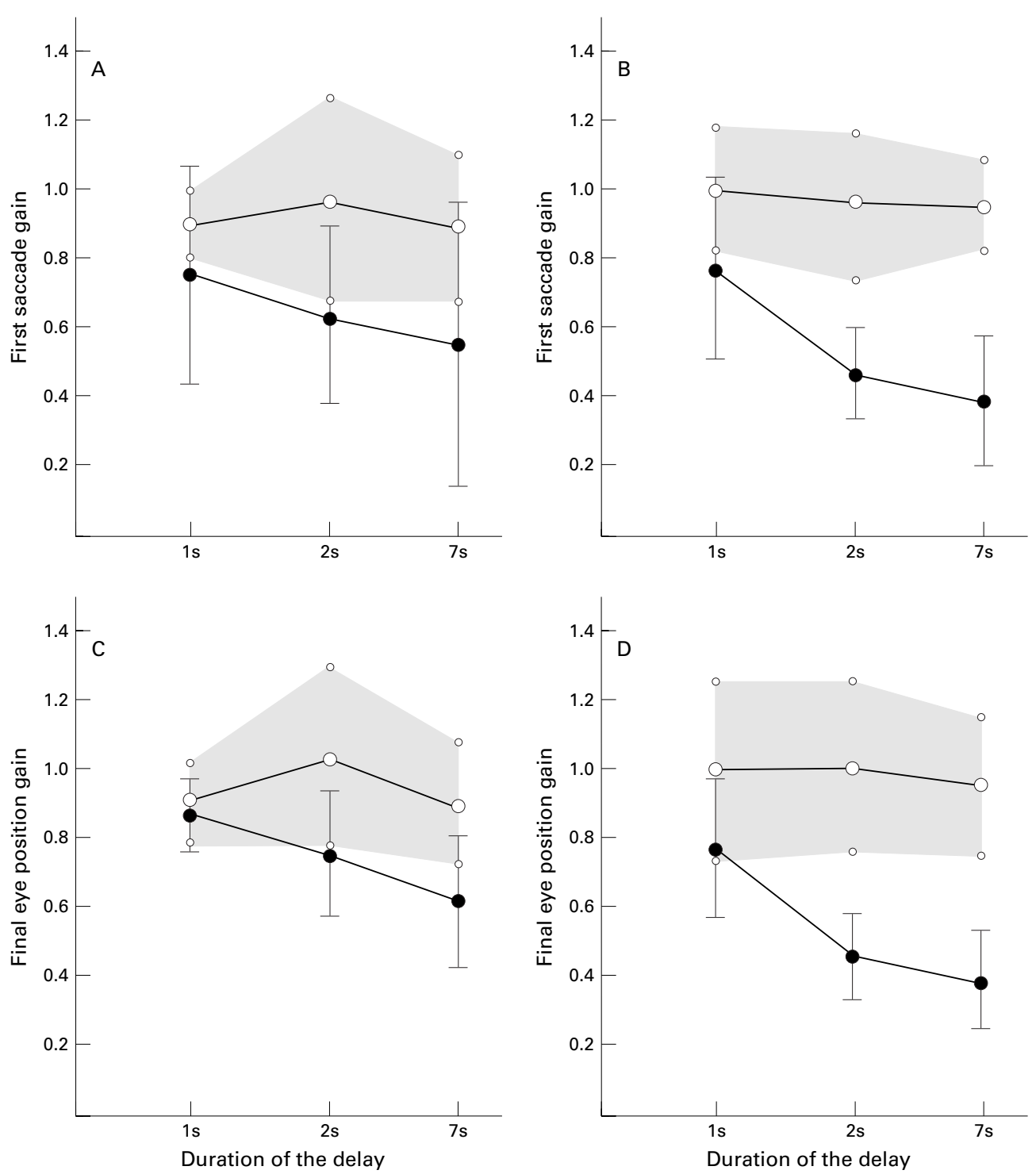

Figure 2 Accuracy of memory guided saccades of patient and controls. $A$ and $C=l e f t ; B$ and $D=$ right. Closed circles = patient; open circles =controls. Note the marked delay dependent decrease in the gain of rightward memory guided saccades for both first saccades and final eye positions. Leftward memory guided saccades were less affected.

gain and rightward and leftward final eye position gain with a $1 \mathrm{~s}$ delay. In the control group, mean gains of first saccades and final eye positions were independent of the memorisation delay (fig 2).

In the memory guided pointing task, the patient's rightward gain was 1.06 , within $2 \mathrm{SD}$ of controls (0.81-1.21) and the leftward gain was 1.15 , thus slightly above $2 \mathrm{SD}$ of controls (0.94-1.10).

\section{Discussion}

Our patient had a lesion involving mainly the body of the left CN. Another damaged structure was the posterior part of the left putamen. A similar but smaller lesion also existed in the right hemisphere. Impairments of several ocular motor parameters were found, with in particular a markedly decreased gain of rightward memory guided saccades. This impairment was unlikely to be due to a perceptual or a motor deficit since rightward visually guided saccades were only slightly impaired.
Furthermore, the absence of improvement in saccade accuracy between first saccade and final eye position argues against a pure motor deficit. The delay dependent increase in inaccuracy of memory guided saccades shows that our patient could not maintain accurate spatial information over several seconds and thus suggests a spatial short term memory deficit. However, memory guided pointing was normal rightwards and slightly abnormal leftwards. An additional experiment in our patient with memory guided pointing after a 1 second delay yielded values identical to those at 7 second delay, thus arguing against a left spatial memory deficit in the pointing task. Normal neurological examination in our patient was in striking contrast to severe cognitive impairments, such as loss of psychic autoactivation and reduced verbal fluency, as has previously been described in patients with bilateral lesions involving the head of the caudate nucleus. ${ }^{13-15}$ These apparently conflicting results may be explained by the existence of 
a functional compartmentalisation of the $\mathrm{CN}$ that reflects the topography of prefrontostriatal connections. ${ }^{16}$ In the monkey, the orbitofrontal cortex and the dlPFC, involved in spatial memory, project mainly to the head of the $\mathrm{CN},{ }^{17}$ whereas the arcuate region, corresponding to the FEF, is connected mainly with the body of the $\mathrm{CN}$ and the putamen. ${ }^{18}$ Therefore, normal accuracy in the memory guided pointing tasks can be explained by the integrity of the head of the $\mathrm{CN}$, and the impairment of memory guided saccades by damage to the body of the CN.

In the monkey, electrophysiological studies have shown that the $\mathrm{CN}$ and the $\mathrm{SNpr}$ contain several neuronal populations with saccade related activity, most of them being context dependent. ${ }^{19}$ Some neurons are active before visually guided saccades, but most neurons are active during memory guided saccades, remaining silent or weakly activated in the context of spontaneous or visually guided saccades. ${ }^{7-9}$ Among these neurons, some are activated immediately before memory guided saccade initiation, and others during the delay. ${ }^{7-9}$ All of them show a strong preference for contraversive saccades. Furthermore, and by contrast with neurons in the dlPFC, the encoded signal seems to represent the metrics of the forthcoming memory guided saccade rather than visuospatial input. ${ }^{9}$

A unilateral dopaminergic depletion of the $\mathrm{CN}$, by local injection of 1-methyl-4-phenyl-12-3-6-tetrahydropyridine, affects accuracy of visually guided saccades and markedly impairs memory guided saccades. ${ }^{20}$ Our data are consistent with these results. The bilateral, although predominantly leftward memory guided saccade hypometria, dependent on the duration of the delay, was consistent with the larger right sided $\mathrm{CN}$ lesion. The lesser impairment of visually guided saccades probably reflects the relatively fewer number of neurons discharging before visually guided saccades in the body of the CN. ${ }^{9}$ An impairment in accuracy of memory guided saccades is also seen after lesions of the dlPFC and/or the FEF. $^{51112}$ However, by contrast with the former ${ }^{11}$ and similarly to the latter, ${ }^{12}$ our patient performed normally in the antisaccade task. Moreover, after FEF lesions, latency of all types of voluntary saccades is increased, ${ }^{12}$ whereas in our patient latencies of visually guided saccades and antisaccades were normal and a selective increase in memory guided saccade latency was found. ${ }^{12}$
In conclusion, the results of our patient together with previously reported experimental findings suggest that the body of the $\mathrm{CN}$ belongs to a corticosubcortical network controlling short term spatial memory especially devoted to eye movements.

1 Fuster JM. The prefrontal cortex and its relation to behaviour. Prog Brain Res 1991;87:201-11.

2 Friedman HR, Goldman-Rakic PS. Coactivation of prefrontal cortex and inferior parietal cortex in working memory tasks revealed by $2 \mathrm{DG}$ functional mapping in the rhesus monkey. F Neurosci 1994;14:2775-88

3 Funahashi S, Bruce CJ, Goldman-Rakic PS. Dorsolateral prefrontal lesions and oculomotor delayed-response performance: evidence for mnemonic "scotomas". $\mathcal{F}$ Neurosci 1993;13:1479-97.

4 Bruce CJ, Goldberg ME. Primate frontal eye fields. I. Single neurons discharging before saccades. I Neurophysiol 1985;53:603-35.

5 Funahashi S, Bruce CJ, Goldman-Rakic PS. Neuronal activity related to saccadic eye movements in the monkey's dorsolateral prefrontal cortex. F Neurophysiol 1991;65: 1464-97.

6 Constantinidis C, Steinmetz MA. Neuronal activity in posterior parietal area $7 \mathrm{a}$ during the delay periods of a spatial memory task. F Neurophysiol 1996;76:1352-5.

7 Hikosaka O, Wurtz RH. Visual and oculomotor functions of monkey substantia nigra pars reticulata. III. Memorycontingent visual and saccade responses. I Neurophysiol 1983;49:1268-84

8 Hikosaka O, Sakamoto M, Usui S. Functional properties of monkey caudate neurons. II. Visual and auditory responses. F Neurophysiol 1989;61:799-813.

9 Hikosaka O, Sakamoto M, Usui S. Functional properties of monkey caudate neurons. I. Activities related to saccadic eye movements. F Neurophysiol 1989;61:780-98.

10 Hikosaka O, Sakamoto M, Usui S. Functional properties of monkey caudate neurons. III. Activities related to expectation of target and reward. F Neurophysiol 1989;61:814-32.

11 Pierrot-Deseilligny C, Rivaud S, Gaymard B, et al. Cortical control of memory-guided saccades in man. Exp Brain Res 1991;83:607-17.

12 Rivaud S, Müri RM, Gaymard B, et al. Eye movement disorders after frontal eye field lesions in humans. Exp Brain Res 1994;102:110-20.

13 Mrabet A, Mrad-Ben Hammouda I, Abroug Z, et al. Infarctus bilatéral des noyaux caudés. Rev Neurol (Paris) 1994;150:67-9.

14 Rodier G, Tranchant C, Mohr M, et al. Neurobehavioral changes following bilateral infarct in the caudate nuclei: a case report with pathological analysis. I Neurol Sci 1994;126:213-8.

15 Pickett ER, Kuniholm E, Protopapas A, et al. Selective speech motor, syntax, and cognitive deficits associated with bilateral damage to the putamen and the head of the caudate nucleus: a case study. Neuropsychologia 1998;36: $173-88$

16 Alexander GE, Crutcher MD. Functional architecture of basal ganglia circuits: neural substrates of parallel processing. Trends Neurosci 1990;13:266-71.

17 Yeterian EH, Pandya DN. Prefrontostriatal connections in relation to cortical architectonic organization in rhesus monkeys. F Comp Neurol 1991;312:43-67.

18 Stanton GB, Goldberg ME, Bruce CJ. Frontal eye field efferents in the macaque monkey: I. Subcortical pathways and topography of striatal and thalamic terminal fields. $\mathcal{F}$ Comp Neurol 1988;271:473-92.

19 Wurtz R H, Hikosaka O. Role of the basal ganglia in the initiation of saccadic eye movements. Prog Brain Res 1986;64: $175-90$.

20 Kori A, Miyashita N, Kato M, et al. Eye movements in monkeys with local dopamine depletion in the caudate nucleus. II. Deficits in voluntary saccades. F Neurosci 1995 ; 15:928-41. 